\title{
A rare case of cavernous hemangioma of maxillary sinus
}

\section{Introduction}

Hemangiomas of the sinonasal tract are rare and because these lesions lack the typical signs or symptoms, they can be confused with other malignant conditions. We report a case of cavernous hemangioma of the maxillary sinus in a 9 yrs old girl that was started on beta blocker. Although computed tomography (CT) showed enhancing areas within the mass, the substantial bone erosion and remodeling made it difficult to differentiate this cavernous hemangioma from other expansile maxillary sinus lesions. We present the CT findings of this lesion and discuss the differential diagnoses and potential therapeutic approaches.

Although hemangiomas are relatively common in the head and neck, they occur infrequently in the sinonasal cavity, ${ }^{1-4}$ It is difficult to differentiate hemangiomas from more common expansile maxillary sinus lesions on the basis of the patient's symptoms and imaging findings. We report a case of maxillary sinus hemangioma and we discuss the imaging features, which may suggest the diagnosis.

\section{Case presentation}

A 9 years old girl, presented with longstanding, left sided nasal obstruction and serosanguinous nasal discharge. She had been experiencing nasal obstruction and intermittent epistaxis for one year already. Her past medical history was otherwise unremarkable. The patient on anterior rhinoscopy, a bulge was detected, arising from the left nasal wall and impinging on the septum. The computed tomography (CT) examination was performed on a spiral scanner. The CT scan obtained following intravenous contrast administration revealed a large, inhomogeneously enhancing mass in the left maxillary sinus and nasal cavity (Figure 1-3). The nasal turbinates and the medial wall of the maxillary sinus had been eroded by the mass. There was also a

\author{
Volume 3 Issue 2 - 2016
}

\author{
Magid Althbety, Ziyad Alzahim, Ahmad \\ Albanna,Waleed Al-Manie, Lulu Albahlal \\ Department of ORL \& HNS, Security Forces Hospital, Saudi \\ Arabia
}

Correspondence: Magid Althbety, Department of Head \& Neck Surgery, Security Forces Hospital, Riyadh, Saudi Arabia, Tel 009665549990 47,Email msb-althobaiti@hotmail.com

Received: APRIL I4, 2016 | Published: May 26, 2016

bony remodeling in the anterior wall of the maxillary sinus. Because of the suspicion of sinonasal cancer, a biopsy was taken to obtain tissue from the nasal cavity and maxillary sinus. However, the biopsy revealed multiple submucosal dilated vessels filled with blood, fibrin deposition and organized thrombi stain for fungal organism negative, findings consistent with hemangioma. The microscopic examination showed dilated sinusoidal vascular channels in the stroma lining with an interconnecting fibrous wall covered with flat endothelium. The Lumina of these spaces were filled with blood. The histologic features were most compatible with cavernous hemangioma (Figure 4). On follow up, patient improvement regarding nasal obstruction and less nasal bleeding.

After less than 2 month since pediatric cardiologist started her on beta-blocker (Propranolol) $1 \mathrm{mg} / \mathrm{kg} /$ day, three times a day, dose increased 2-3 mg/kg/day, will be continued for 6-12 months. With weight gain adjusted on monthly basis.

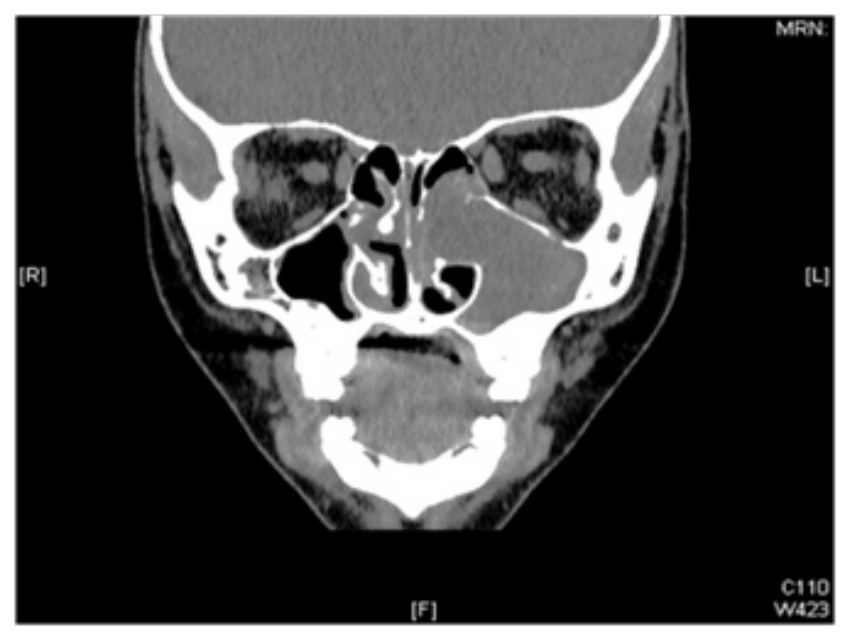

Figure I Coronal CT scan showing a large expansile lesion occupying mainly the left maxillary antrum with distended and thinned out bony wall of the left maxillary cavity, the lesion extends to the left nasal cavity and ethmoidal air cells with partial obliteration of the left nasal cavity. 


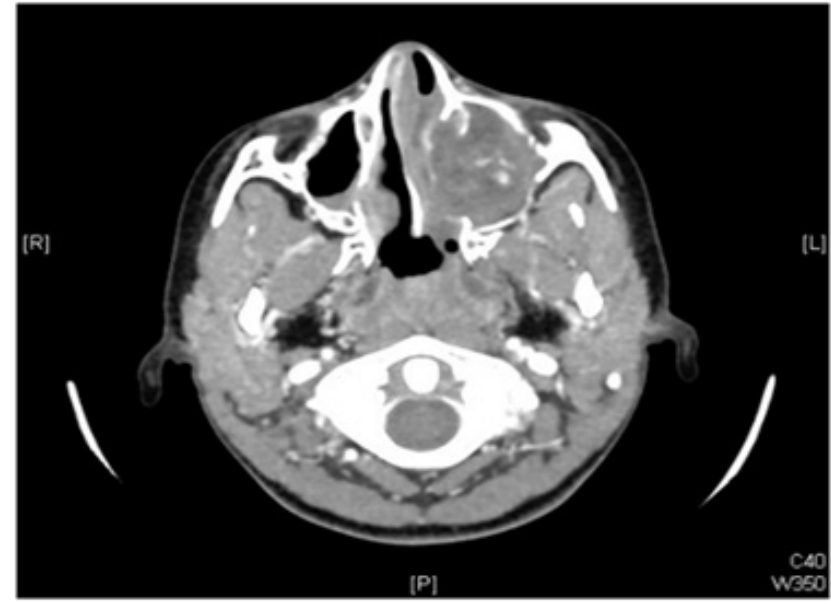

Figure 2 Axial CT scan showing large, inhomogeneously enhancing mass in the left maxillary sinus and nasal cavity. The nasal turbinates and the medial wall of maxillary sinus had been eroded by the mass.

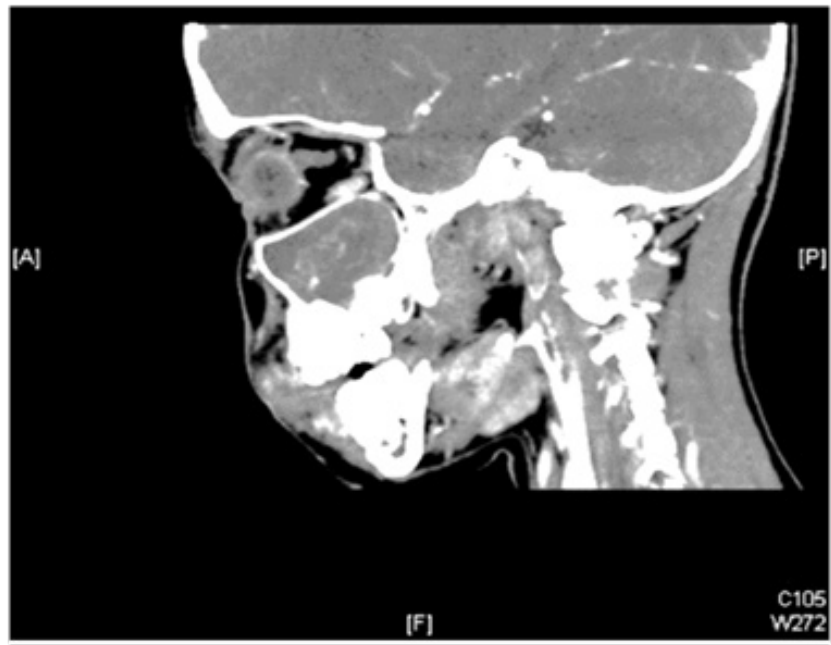

Figure 3 Sagittal CT revealed a large expansile mass occupying the whole left maxillary sinus

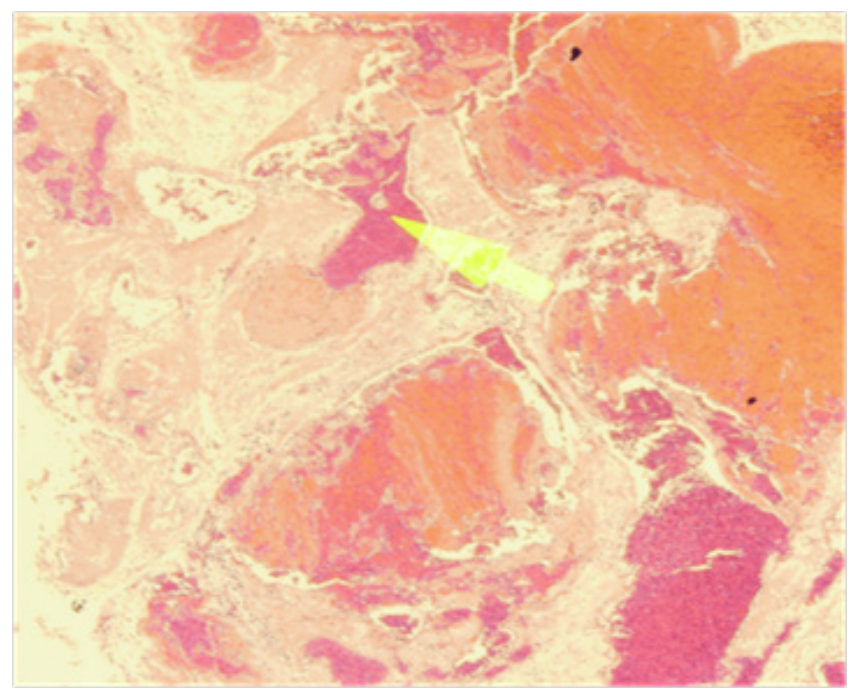

Figure 4 Multiple sub mucosal dilated vessels filled with blood, fibrin deposition and organized thrombi. Stain for fungal organism is negative. Findings consistent with Hemangioma "Cavernous Hemangioma". 


\section{Discussion}

Hemangiomas of the head and neck are benign vascular lesions, and although they are common in these locations, they occur infrequently in the paranasal sinuses. Hemangiomas are divided into capillary and cavernous types, depending on the dominant vessel size seen on microscopic examination. Capillary hemangioma is the more common type and is composed of capillary-sized vessels lined with flattened epithelium. Cavernous hemangiomas are composed of large, endothelium-lined vascular spaces. The common symptoms of the sinonasal hemangiomas include nasal obstruction, epistaxis and occasionally a visible nasal mass. Although there have been multiple clinical studies of nasal hemangioma, only less than 20 cases of maxillary hemangioma have been reported.

The radiographic appearance of hemangiomas has been reported in the literature. Previously reported CT features of maxillary sinus hemangioma can be summarized as depicting a highly vascularized, soft-tissue mass. Although several enhancing portions have been noted on CT scans, even larger areas did not enhance because of necrosis and hemorrhage within the tumor. Furthermore, Hemangiomas have been reported to cause changes in adjacent bone. According to Dillon et al., ${ }^{5}$ capillary Hemangiomas of the nasal vault had benign appearing bone changes, consisting of remodeling or expansion. Kim et al., ${ }^{6}$ reported two cases of cavernous hemangioma that caused erosion of the wall of the maxillary sinus, nasal turbinate and orbit. According to Weiss et al., amorphous or curvilinear calcification is a non-specific finding, whereas phlebolith formation is a more specific finding of cavernous hemangioma. In our case, CT also showed a soft tissue mass, which caused expansile bone remodeling of the maxillary sinus, with a small calcification within the lesion, similar to previously reported CT findings. We therefore, assume that this CT finding offers a diagnostic clue regarding the presence of a hemangioma. According to Itoh et al. MR images of cavernous hemangioma reflect the signal of unclotted blood and they have prolonged $\mathrm{T} 1$ and $\mathrm{T} 2$ relaxation times, which result in low signal intensity on T1-weighted images and high signal intensity on T2-weighted images. However, these lesions do not show any of the signal voids associated with the hypervascularity, often seen in other types of vascular malformation. Dillon et al., ${ }^{5}$ reported that, on T2-weighted sequences, capillary hemangioma of the nasal vault shows a hypointense rim surrounding a mass of mixed signal intensities. Pathology examination revealed that these signals were apparently correlated with areas of clotted blood.

Organized hematoma $(\mathrm{OH})$ is the most difficult lesion to differentiate, both clinically and radiologically, from sinonasal cavernous hemangioma. Although Yagisawa et al. suggested that hemangioma and sinonasal $\mathrm{OH}$ are the same pathologic entity; the fact that the vascular Lumina of cavernous hemangioma are usually larger than those of $\mathrm{OH}$ on histologic examination still raises the question of the probability of the different nature of the two lesions. In addition to $\mathrm{OH}$, mucocele, fungus ball, inflammatory polyp, cholesterol granuloma, inverted papilloma, and carcinoma can present as an expansile mass. Contrast material administration is useful because mucocele, fungus ball, polyp and cholesterol Granuloma are not usually enhanced. Carcinoma has a pattern of nodular enhancement.
The surgical resection is the main treatment for maxillary sinus hemangioma. According to tumor extent, different surgical resection methods can be used for maxillary sinus hemangioma, such as lateral rhinotomy, the Caldwell-Luc operation or endoscopic sinus surgery. Preoperative transarterial embolization can decrease the tumor size and reduce the risk of hemorrhage during surgery. ${ }^{9-12}$

\section{Conclusion}

Sinonasal cavernous hemangioma may be mistaken for a locally aggressive neoplasm. The $\mathrm{CT}$ and MR images have demonstrated an expansile, well-enhanced heterogeneous mass, with bone remodeling and erosive change. Internal calcification may also be visible. Although it is a very unusual tumor, based on the imaging findings, cavernous sinus hemangioma should be considered in the differential diagnosis of a paranasal sinus tumour.

\section{Acknowledgements}

None.

\section{Conflicts of interest}

The author declares there is no conflict of interest.

\section{References}

1. Raboso E, Rosell A, Plaza G, et al. Haemangioma of the maxillary sinus. J Laryngol Otol. 1997;111(7):638-640.

2. Iwata N, Hattori K, Nakagawa T, et al. Hemangioma of the nasal cavity:a clinicopathologic study. Auris Nasus Larynx. 2002;29(4):335-339.

3. Jammal H, Barakat F, Hadi U. Maxillary sinus cavernous hemangioma:a rare entity. Acta Otolaryngol. 2004;124(3):331-333.

4. Kanazawa T, Inoue R, Ohta Y, et al. Maxillary haemangioma successfully resected by endoscopic approach. J Laryngol Otol. 2009;123(7):793795.

5. Dillon WP, Som PM, Rosenau W. Hemangioma of the nasal vault:MR and CT features. Radiology. 1991;180(3):761-765.

6. Kim HJ, Kim JH, Kim JH, et al. Bone erosion caused by sinonasal cavernous hemangioma:CT findings in two patients. Am J Neuroradiol. 1995;16(5):1176-1178.

7. Weiss SW, Enzinger FM, Goldblum JR. Enzinger and weiss's soft tissue tumors. ( $5^{\text {th }}$ edn $)$, USA; Mosby Louis, 2008.

8. Itoh K, Nishimura K, Togashi K, et al. MR imaging of cavernous hemangioma of the face and neck. J Comput Assist Tomogr. 1986;10(5):831835 .

9. Song CE, Cho JH, Kim SY, et al. Endoscopic resection of haemangiomas in the sinonasal cavity. J Laryngol Otol. 2009;123(8):868-872.

10. Yagisawa M, Ishitoya J, Tsukuda M. Hematoma-like mass of the maxillary sinus. Acta Otolaryngol. 2006;126(3):277-281.

11. Lee HK, Smoker WR, Lee BJ, et al. Organized hematoma of the maxillary sinus:CT findings. AJR Am J Roentgenol. 2007;188(4):W370373.

12. Som PM, Branwein MS, Kassel EE. Tumors and tumor like conditions of the sinonasal cavities. ( $5^{\text {th }}$ edn $)$; USA, Mosby Louis, 2011. 